\title{
Publicaciones científicas: una estrategia de internacionalización de las universidades
}

\author{
Scientific Publications: an internationalization strategy for the universities
}

\author{
Juan Asdrúbal Flores-Pacheco ${ }^{1}$ \\ Jhonny Francisco Mendoza ${ }^{2}$
}

\section{Resumen}

$\mathrm{Al}$ analizar la calidad de una Institución de Educación Superior es imprescindible evaluar el impacto y la visibilidad de su producción de conocimientos basados en la mejora continua de la ciencia. Sin embargo, la producción científica no está siendo divulgada debidamente lo que se traduce en la reducción significada de los beneficios académicos e institucionales que esto trae. En el marco de la nueva normalidad la internacionalización desde casa, toma auge al tiempo que se adoptan nuevas estrategias para potencializar las fortalezas de las universidades. En este artículo de opinión se analiza esta realidad, en particular para las universidades comunitarias de la Costa Caribe de Nicaragua.

Palabras clave: Visibilidad; impacto; Investigación; Desarrollo e innovación.

\section{Abstract}

When analyzing the quality of a Higher Education Institution, it is essential to evaluate the impact and visibility of its production of knowledge based on the continuous improvement of science. However, scientific production is not being properly disclosed, which translates into a significant reduction in the academic and institutional benefits that this brings. Within the framework of the new normal, internationalization from home is booming while new strategies are adopted to enhance the strengths of universities. This opinion article analyzes this reality, in particular for the community universities of the Caribbean Coast of Nicaragua.

Keywords: Visibility; Impact; Research; Development and Innovation

1 Doctor en Conservación y Uso Sostenible de Sistemas Forestales, Director de Investigación y Posgrado, Bluefields Indian \& Caribbean University (BICU), email: asdrubal.flores@do.bicu.edu.ni; https://orcid.org/oooo-ooo1-6553-7202

2 Máster en Investigación aplicada a la educación, docente tiempo completo Facultad de Ciencias de la Educación y Humanidades, Bluefields Indian \& Caribbean University (BICU), e-mail: jhonny.mendoza@bicu.edu.ni | https://orcid.org/oooo-ooo1-7634-5601

Recibido: 08/01/2021 - Aprobado: 05/02/2021 


\section{Introducción}

Es evidente el interés de las Instituciones de Educación Superior (IES) de Nicaragua en el aumento de la producción científica por parte de la comunidad universitaria. En este sentido, no sólo se debe impulsar la producción científica estudiantil por medio de la realización de diversas modalidades de culminación de estudios con fines de graduación que se traduzcan en documentos con potencial publicable (Mamani-Benito, 2020), sino, principalmente de los docentes que, además de su rol de educadores, la tarea investigativa es inherente a su profesión por lo que deben considerarse investigadores por defecto (Hernández Arteaga, 2009).

Es imprescindible detallar los beneficios que trae esta actividad tanto para el autor y la institución académica a la que éste representa y que le respalda (Fernando García; 2008) Si bien es cierto en primer momento se piensa únicamente en el prestigio profesional del investigador o grupo que realiza la investigación; luego se piensa en la posición en la que se encuentra la revista donde se ha logrado posicionar el artículo, es decir, el factor de impacto con el que diversas bases de datos y editoriales categorizan la calidad de las revistas y que indudablemente ayudan mucho al aumento del rendimiento de la producción científica de estos profesionales (Martin, 2020).Sin embargo, se comete el error de pensar que las publicaciones son únicamente para el aumento del índice de impacto (índice h e índice i1o) de los investigadores (Pacheco - Mendoza, 2018),sino también de la visibilidad de las instituciones académicas como una herramienta de proyección e internacionalización que permita no sólo la difusión de su trabajo, sino, otros beneficios propios de este proceso (Arias, 2020).

\section{Desarrollo}

Con cada publicación respaldada por el personal científico y técnico que se realice desde la IES, ambas partes ganan, además de prestigio, escalan más peldaños en el posicionamiento como instituciones que no sólo son copiadoras del conocimiento, sino, generadoras del mismo por medio de sus procesos investigativos e innovadores, nacidos de la necesidades sociales, ambientales y económicas de su entorno real (Díaz \& Pedroza, 2018). Este avance, trae consigo una serie de oportunidades de las cuales destacan el establecimiento de referencias y el fortalecimiento de la hoja de vida profesional e institucional, con ello el reconocimiento en el campo como expertos que a su vez abren las oportunidades de obtener ayudas económicas para la investigación, desarrollo e innovación (I+D+i) (Contreras, et al., 2015). No menos importante, es el posicionamiento en la comunidad científica como profesional e institución de referencia que, además de ser un indicador de calidad, posibilita la oportunidad de pasantías, prácticas y estancias científicas en busca de la profesionalización u obtención de ulteriores grados científicos contribuyendo a la mitigación de la endogamia académica, fenómeno con grandes efectos en el deterioro de la calidad en la enseñanza superior (Meza, 2011). 
Internacionalizar las universidades no debe ser comprendido como el hecho de la creación de portales o páginas web donde se coloquen imágenes de sus campus, del personal y alumnos, algunos contactos y, en un caso, aspirar que algún extranjero residente en el país decida cursar estudios en ellas. Tampoco es aspirar que nuestro personal y estudiantes visiten turísticamente universidades extrajeras y que a su regreso sus aprendizajes no sean vinculantes ni convalidables. Diametralmente, opuesto a esta realidad, la Internacionalización de la Educación Superior debe ser un proceso formativo, sistemático, lógico y dirigido a potencializar las más destacadas capacidades de los individuos que han demostrado capacidad no sólo de trabajo, sino sinérgicas organizativas y que, ante todo, sean proactivos e innovadores, maximizando el aprendizaje formal y el no formal, consistente en la experiencia que no será replicado, sino adaptado y adecuado a necesidades locales. Así, la calidad de la educación superior y la formación académica de docentes y nuevos profesionales, debe adecuarse a la proyección en el ámbito nacional e internacional por medio de las publicaciones científicas (Näslund-Hadley et al., 2012).

En el caso de Nicaragua, como otros países en vías de desarrollo en América Latina y el Caribe, la producción científicas es limitada, no por el hecho de no existir, sino por la inexplicable costumbre de no difundir los resultados de las investigaciones y trabajos técnicos realizados (EDUQUEMOS, 2014). En el país se ha vivido un auge en la especialización en diversos grados que van desde pasantías hasta doctorados que han traído consigo la incorporación de profesionales con elevadas capacidades científicas (CNU, 2016); pero, igual de importante es la experiencia de estos profesionales con una nueva visión de trabajo holístico e integrador en que se ha eliminado el precepto del aislamiento académico siendo sustituido por la colaboración entre individuos e instituciones (Luchilo, 2006).

Aún más, específicamente en el caso de las universidades comunitarias del Caribe nicaragüense, esta postura ha dejado de ser una opción para convertirse en una necesidad, no por el hecho de su simple mención en ámbitos externos, sino como una estrategia de difusión de un modelo incluyente y formativo que respeta y fomenta la identidad cultural de las minorías en tanto se procura la formación profesional al más alto nivel posible (Cassell's,2017).Sin embargo, este es un inicio que, lejos de ser el resultado final, debe ser el punto de partida para un proceso continuo y estable en el que, no sólo se busque el desarrollo de planes educativos, sino también la integración de capacidades y visión en los educadores y educandos. Conclusiones

En una cita celebre de Albert Einstein, se lee: "Locura es hacer lo mismo una y otra vez esperando obtener resultados diferentes"; en ella, se sintetiza el futuro de los profesionales y las instituciones que los albergan sino son capaces de integrarse al mundo del crecimiento y las interacciones entre universidades que, buscan la calidad educativa. En condiciones de limitados recursos humanos y económicos es imprescindible la difusión del trabajo científico técnico realizado en aras de la búsqueda 


\section{EDUCACIÓN}

de aleados que permitan la potencialización del desarrollo y producción científica e innovadora. Es por ello, señores lectores, que las publicaciones técnicas, informativas y especialmente las científicas, no deben continuar considerándose una opción, deben ser vista como herramientas versátiles con capacidad más allá de la divulgativa.

\section{Lista de referencias}

Arias, J. (2020). Proyecto de tesis - Guía para la elaboración (J. Arias (ed.); 10). Biblioteca Nacional del Perú. www.agogocursos.com

Cassell's, R. (2017). El modelo Educativo de la BICU : el caso de los estudiantes afrodescendientes e indígenas en la década 2007-2016 The educational model of the BICU : the case of Afro-descendant and indigenous students in the decade 2007-2016. Revista Científica de FAREM-Estelí. Medio ambiente, tecnología y desarrollo humano., 23(6), 3-20.

Concejo Nacional de Universidades (2016). Rendición de cuentas sociales 2015 (10). Consejo Nacional de Universidades (CNU) de Nicaragua. http://www.cnu. edu.ni/

Contreras, F. G., Buzeta, L. P., \& Pedraja-Rejas, L. (2015). Importancia de las publicaciones académicas: algunos problemas y recomendaciones a tener en cuenta. Idesia (Arica), 33(4), 111-119. https://doi.org/10.4067/So718-34292015000400014

Díaz, A., \&yPedroza, M. (2018). Indicadores de impacto en la investigación científica Indicators of impact on scientific research. Revista Cientifica de FAREM-Estelí: Medio Ambiente, Tecnología y Desarrollo Humano, 7(25), 6o-66. https://doi.org/ http://dx.doi.org/10.5377/farem.voi25.5683

EDUQUEMOS Informe del Foro EducativoNicaragüense. (2014). Informe del proceso educativo Nicaragua. Calidad y Equidad para el Desarrrollo Humano. www. eduquemos.org.ni

Fernández García, J. J. (2008). Más allá de Google. En Infonomia. http://www.infonomia.com/pdf/Mas_alla_de_Google_20o8.pdf

Hernández Arteaga, I. (2009). docente profesionales investigador en la formación de The Teacher- Researcher's Role in Professional Training Le rôle de l' enseignant -chercheur dans la formation des. Revista Virtual Universidad Católica del Norte, 27, 1-21. http://www.redalyc.org/pdf/1942/194215432011.pdf\%5Cnhttp:// revistavirtual.ucn.edu.co/ 
Luchilo, L. (2006). Movilidad de estudiantes universitarios e internacionalización de la educación superior. Revista iberoamericana de ciencia tecnología y sociedad, 3(7), 105-133.

Mamani-Benito, O. J. (2020). Limitada producción científica de la comunidad universitaria: Rol del jurado de tesis. Revista Medica Herediana, 31(2), 134-135. https:// doi.org/10.20453/rmh.v31i2.3779

Martín, S. (2020). ¿Dónde publicar? Methodo Investigación Aplicada a las Ciencias Biológicas, 5(3), 119-124. https://doi.org/10.22529/me.2020.5(3)o9

Meza, U. (2011). Reflexión e investigación - Revista Editorial del Congreso de Eduación Superior (L. Vélaz, J. Miranda, \& J. Alandete (eds.); 10).

Näslund-Hadley, E., Meza, D., Arcia, G., Rápalo, R., \& Rondón, C. (2012). Educación en Nicaragua: Retos y Oportunidades. BID Banco Interamericano de Desarrollo, IDB-TN-458, 32. http://www.iadb.org

Pacheco-Mendoza, J. (2018). ¿Cómo usar SCOPUS ? (p. 38). Pontificia Universidad Católica del Perú 